\title{
3 Research Suare \\ Sphingosine 1-phosphate, a Novel TREM2 Ligand, Promotes Phagocytosis and Reduce Ischemic Injury
}

Xiu-Lan Sun ( $\nabla$ xiulans@njmu.edu.cn )

Nanjing Medical University https://orcid.org/0000-0001-8741-3833

\section{Teng-Fei Xue}

Nanjing Medical University

Juan Ji

Nanjing Medical University

Ruo-Bing Guo

Nanjing Medical University

Yu-Qin Sun

Nanjing Medical University

Xi-Peng Wang

Nanjing Medical University

Wei Guo

Nanjing Medical University

Zhen-Yu Cai

Nanjing Medical University

Xin-Xin Huang

Nanjing Medical University

Jin Yang

Nanjing Medical University

Hong Cheng

Jiangsu Province Hospital and Nanjing Medical University First Affiliated Hospital

Hai-Bin Shi

Jiangsu Province Hospital and Nanjing Medical University First Affiliated Hospital

Research article

Keywords: S1P, TREM2, microglia, phagocytosis, stroke

Posted Date: December 1st, 2020

DOI: https://doi.org/10.21203/rs.3.rs-114600/v1 
License: (c) (i) This work is licensed under a Creative Commons Attribution 4.0 International License. Read Full License 


\section{Abstract}

Background: Activation of TREM2 protects against brain injury in ischemic stroke via immunoregulation. However, the endogenous ligand of TREM2 remains unknown. Here, we tested the hypothesis that S1P, an immunoregulator, functions as TREM2 ligand to promote microglial phagocytosis.

Methods: SD rats, $\mathrm{C} 57 \mathrm{BL} / 6 \mathrm{~J}$ mice and $\mathrm{TREM}^{-/-}$mice were subjected to transient middle cerebral artery occlusion, and primary microglia were subjected to oxygen-glucose deprivation. Phagocytosis was investigated via immunofluorescence and two-photon microscope. LC-MS/MS, microscale thermophoresis and surface plasmon resonance were used to confirm the TREM2-S1P interaction.

Results: FTY720, an analog of S1P, promoted microglial phagocytosis in ischemic stroke independent of S1PRs expressed on microglia. S1P was confirmed to be a novel endogenous ligand for TREM2 and promote cellular debris clearance. The enhanced cellular debris clearance ameliorated neurological score and infarct volume, relying on TREM2. Moreover, FTY720 was demonstrated to promote hemoglobin clearance in intracerebral hemorrhage and ameliorate hemorrhagic injury.

Conlusions: The present work reveals for the first time that S1P acts as a novel endogenous ligand of TREM2 to effectively promote microglial phagocytosis, and provides a new lead compound for developing TREM2 modulator.

\section{Background}

Sphingosine is one of the most important sphingolipid metabolites, named after the Sphinx for its mysterious features. Phosphorylation of sphingosine forms the pleiotropic and bioactive lipid sphingosine-1-phosphate (S1P) (Fig. 1A). Traditionally, S1P acts not only as an intracellular second messenger, but also an extracellular first messenger in both an autocrine and paracrine manner, via binding with S1P receptors (S1PRs) of which there are currently five known subtypes (S1PR1-5)[1-3]. It has been revealed that S1P has a wide range of biological functions including regulating differentiation, survival, proliferation, angiogenesis and immune modulation[4,5]. A few studies have suggested that S1P might regulate microglial phagocytosis[6, 7]. However, the involved mechanisms remain unknown.

As an important phagocytosis mediator, triggering receptor expressed on myeloid cells 2 (TREM2) is a cell surface receptor of the Ig superfamily. It consists of an ectodomain, a transmembrane region and a short cytoplasmic tail, which transmit downstream signal by coupling with DNAX-activating protein of $12 \mathrm{kDa}$ (DAP12)[8, 9]. TREM2 is expressed on osteoclast, macrophage, dendritic cell, and exclusively on microglia in the brain, primary participates in phagocytosis[10-13]. Till now, the endogenous ligand of TREM2 has not been found. However, a diverse set of potential TREM2 ligands have been proposed, such as bacteria, poly-anionic[14, 15], and phospholipids[16].

Stroke is the rapid development of a focal neurologic deficit caused by a disruption of blood supply to the corresponding area of brain and can either be ischemic $(\sim 87 \%)$ or hemorrhagic $(\sim 13 \%)$ [17]. Ischemic 
stroke is a leading cause of mortality and disability without efficient therapeutic strategy, despite thrombolysis and thrombectomy in the acute phase[18, 19]. Disruption of the regional blood supply initiates ischemic cascade leading to neuronal dysfunction and subsequent death[20,21]. Brain edema and inflammation response in the sub-acute phase, contribute to the secondary injury[20,22]. The damaged and dead neurons could release nucleic acid, protein and lipid, to induce neuroinflammation and exacerbate damage[23]. In the intracerebral hemorrhagic stroke, blood products introduced from hematoma such as hemoglobin and iron can exacerbate neuronal death[24, 25]. Thus, promoting phagocytic clearance of neurotoxic cellular debris is beneficial to recovery after stroke and could serve as a promising therapeutic strategy.

In the present work, we confirmed the pro-phagocytic function of S1P and a S1P analog, FTY720, that was not mediated by S1PRs. Because S1P belongs to phospholipids and as well regulates microglial phagocytosis, we speculated and used computer simulation of molecular docking to predicate that S1P might bind to TREM2. Moreover, we verified that S1P and FTY720p could bind to TREM2, promote microglial phagocytosis, and thereby exert neuroprotection in ischemic and hemorrhagic stroke.

\section{Methods}

\section{Animal model and experimental protocol}

Male Sprague Dawley (SD) rats (260 g $\pm 10 \mathrm{~g}$, Animal Core Facility of Nanjing Medical University), C57BL/6J mice (20-25 g, Animal Core Facility of Nanjing Medical University) and TREM2 ${ }^{-/-}$mice (20$25 \mathrm{~g}$, Cyagen Biosciences) were maintained with ad libitum access to standard fodder and water in a well-ventilated environment with approximately $25^{\circ} \mathrm{C}, 50 \% \sim 60 \%$ humidity and a standard $12 \mathrm{~h} \mathrm{light/dark}$ cycle. Animals were assigned to Sham group, model group and FTY720 treated group randomly. Focal cerebral ischemic stroke was induced as described previously[26] and rats were intraperitoneally injected with FTY720 (2 mg/kg, selleck chemicals) or normal saline daily for $24 \mathrm{~h}, 48 \mathrm{~h}$. The ICH modeling was as previously described with slight modification[27]. In brief, a burr hole was made after anesthesia and autologous blood $(10 \mu \mathrm{l})$ injected at a rate of $1 \mu \mathrm{l} / \mathrm{min}$ using a 26 -gauge needle at the coordinates: $0.2 \mathrm{~mm}$ anterior, $2.5 \mathrm{~mm}$ lateral, and $3.5 \mathrm{~mm}$ ventral to the bregma. The needle was removed $20 \mathrm{~min}$ after injection to prevent reflux. Mice were intraperitoneally injected with FTY720 $(1 \mathrm{mg} / \mathrm{kg})$ or normal saline daily for $72 \mathrm{~h}$. The protocols were approved by the Institutional Animal Care and Use Committee of Nanjing Medical University.

\section{TTC staining}

Brains were removed and cut into cerebral coronary slices of $2 \mathrm{~mm}$ at $48 \mathrm{~h}$ after surgery, which were incubated in TTC $\left(2,3,5\right.$-Triphenyltetrazolium chloride, $1 \%$, sigma) for 5 min at $37^{\circ} \mathrm{C}$ and then, placed in $4 \%$ paraformaldehyde solution ( $\mathrm{pH} \mathrm{7.4)}$ at $4{ }^{\circ} \mathrm{C}$ to fix up overnight. Photographies were taken and infarct size was analyzed by ImageJ software.

\section{Neurological Deficit Scoring}


Neurological deficit of MCAO rats was classified and scored referring to Bederson's scale scores at $24 \mathrm{~h}$, $48 \mathrm{~h}$ after surgery. When following conditions occured, corresponding rats were excluded and supplemented: 0 score, massive bleeding during surgery, postoperative respiratory abnormality, early mortality and subarachnoid hemorrhage.

Neurological deficit of ICH mice were assessed at 24 h, 48 h, $72 \mathrm{~h}$ after ICH. All mice were scored on six neurologic tests, including body symmetry, gait, climbing, circling behavior, front limb symmetry, compulsory circling and whisker response[28]. Each test was graded from 0 to 4, establishing a maximum deficit score of 28.

\section{Immunofluorescence and two-photon microscope}

After anesthetizing, rats were transcardially perfused with $37^{\circ} \mathrm{C}$ saline followed by $4 \%$ paraformaldehyde (PFA). The brains were removed and postfixed in $4 \%$ PFA for $24 \mathrm{~h}$ to $48 \mathrm{~h}$ at $4{ }^{\circ} \mathrm{C}$. Then, they were embeded in paraffin and sectioned coronally at an interval of $5 \mathrm{~mm}$. Coronal sections were processed sequently as following steps: paraffin melting for $60 \mathrm{~min}$ at $60^{\circ} \mathrm{C}$, deparaffinage and rehydration, endogenous peroxidase inactivation with $3 \% \mathrm{H}_{2} \mathrm{O}_{2}$ at room temperature for 20 min and washing with $0.01 \mathrm{M}$ PBS for 3 times. After washing, and $0.01 \mathrm{M}$ citrate buffer solution was heated to $92 \sim 98^{\circ} \mathrm{C}$ with microwave oven simultaneously, slices were placed into the solution for retrieval for $15 \mathrm{~min}$ and taken out to cool down naturally to room temperature. For the sake of blocking non-specific antigen, slides were incubated with $10 \%$ normal goat serum for $1 \mathrm{~h}$ at room temperature. Primary antibodies were incubated overnight at $4{ }^{\circ} \mathrm{C}$ at the following dilutions: monoclonal rabbit anti-lba1 (1:500, Wako), monoclonal mouse anti-NeuN (1:400, Millipore), monoclonal mouse anti-CD68 (1:200, Dako) and monoclonal mouse anti-Hb (1:200, abcam), followed by incubation with secondary antibody: Alexa Fluor-488-goat antimouse, Alexa Fluor-546-donkey anti-rabbit, Alexa Fluor-555-donkey anti-goat, Alexa Fluor-647-goat antirabbit (Invitrogen, NY, USA) at a 1:1000 dilution for $1 \mathrm{~h}$. After washing, coronal sections or cells were counterstained with Hoechst 33342 for 15 min and washed thrice with PBS for 5 min each time. The stained sections or cells were visualized and photographed using fluorescent microscope (Zeiss Axio Vert A1) or two-photon microscope (Zeiss LSM880 with NLO \& Airyscan).

\section{Primary microglia and neuron co-culture system}

Primary microglial and neuron cultures were performed as previously described and were isolated from 1to 2-day-old postnatal Sprague-Dawley rats. All of the animal operational procedures were performed in accordance with the Institution for Animal Care and Use Committee and approved by Animal Core Facility of Nanjing Medical University. Briefly, primary cultures of glial cells were obtained from the cerebral cortices, which were earlier digested by $0.25 \%$ trypsin/EDTA (Gibco, Grand Island, NY, USA) at $37^{\circ} \mathrm{C}$ for $10 \mathrm{~min}$ and seeded into poly-d-lysine-coated $\left(0.1 \mathrm{mg} / \mathrm{ml}\right.$; Sigma Chemical, St. Louis, MO, USA) $25-\mathrm{cm}^{2}$ culture flasks. The microglia cultures were maintained for 7 days in Dulbecco's modified Eagle's medium (DMEM) (Gibco) supplemented with 10\% heat-inactivated fetal bovine serum (FBS) (Gibco) at $37^{\circ} \mathrm{C}$ in a humidified $5 \% \mathrm{CO}_{2}-95 \%$ air atmosphere. The neuron cultures were maintained in Neuralbasal (Gibco) supplemented with $2 \%$ B27 (Invitrogen) for 6-7 days. 
For in vitro experiments, microglial cells were separated from the mixed primary culture by flapped for $15 \mathrm{~min}$ and then plated $\left(2 \times 10^{4}\right.$ cells per well $)$ in neuron $\left(2 \times 10^{5}\right.$ cells per well in a 24-well plate) culture vessels with DMEM containing 10\% FBS:Neuralbasal $=1: 3$. The cells were co-cultured for further treatment the following day.

\section{Transfection: knock down of TREM2}

Microglia cells in the co-culture system or in culture vessels $\left(6 \times 10^{5}\right.$ cells per well in a 6 -well plate) were transfected using siRNA-mate (Genepharma, Shanghai, China) according to the manufacturer's instructions.

\section{Phagocytosis assay}

TREM2-DAP12 cDNA was generated as previously described[13]. CHO cells were transfected with the construct to generate a stable cell line that express TREM2-DAP12 chimera. The parental cells or transfected $\mathrm{CHO}$ cells were seeded in 24-well plates at the density of $1 \times 10^{4}$ cells per well and incubated overnight. After two washes with PBS, cells were dyed with $5 \mathrm{mM}$ Cell Tracker ${ }^{\mathrm{TM}}$ Green (Thermo scientific) for $20 \mathrm{~min}$, followed by washing and incubation in Opti-MEM medium containing $3 \mu \mathrm{l} / 100 \mu \mathrm{l}$ of pHrodo Red zymosan bioparticles and/or $20 \mu \mathrm{M} \mathrm{S1P}$ and/or $10 \mu \mathrm{g} / \mathrm{ml} \mathrm{LPS}$, or S1P and $2 \mu \mathrm{M}$ CytoD. The treated cells were examined at $2 \mathrm{~h}$ and $4 \mathrm{~h}$ by fluorescent microscope.

\section{Oxygen and glucose deprivation/Reperfusion (OGD/R)}

To initiate OGD, the culture medium was removed, rinsed with phosphate buffered saline (PBS) and replaced with Opti-MEM (Gibco). The cultured cells were placed into the hypoxia chamber (Thermo scientific) with a premixed gas $\left(1 \% \mathrm{O}_{2}, 94 \% \mathrm{~N}_{2}, 5 \% \mathrm{CO}_{2}\right)$ at $37^{\circ} \mathrm{C}$ for $3 \mathrm{~h}$. After OGD, the cells were perfused by $10 \%$ FBS-DMEM medium or $10 \%$ FBS-DMEM medium : Neurobasal $=1: 3$ and transferred to a $5 \% \mathrm{CO}_{2}-95 \% \mathrm{O}_{2}$ air incubator for relative time. Control cells were incubated under normal conditions throughout the procedure.

\section{LC-MS/MS}

BV2 cells were grown to $80 \%-90 \%$ confluency in two dishes and treated with $20 \mu \mathrm{M} \mathrm{S1P}$ or not. After $2 \mathrm{~h}$, the cells were washed thrice with PBS and collected with $400 \mu$ l homogenate buffer per dish $(250 \mathrm{mM}$ sucrose, $10 \mathrm{mM}$ HEPES, $1 \mathrm{mM}$ EDTA, $1 \mathrm{mM}$ DTT, $\mathrm{NaOH}, \mathrm{pH}$ to 7.4). The collected cells were frozen at -80 ${ }^{\circ} \mathrm{C}$ and underwent 5 freezing and thaw cycles to facilitate lysis. Then the buffer was homogenized further with bead mill. After centrifugation at $12000 \mathrm{rpm}$ for $15 \mathrm{~min}$ at $4{ }^{\circ} \mathrm{C}$, the protein of cell lysates was acquired and incubated with $3 \mu \mathrm{l}$ anti-TREM2 (abcam, ab125117) overnight at $4{ }^{\circ} \mathrm{C}$ on a rotating device, followed by adding $100 \mu \mathrm{l}$ protein $\mathrm{A}+\mathrm{G}$ beads/ml lysate overnight to capture the conjugated polymersat 4 ${ }^{\circ} \mathrm{C}$ on a rotating device. Immunoprecipitates were collected by centrifugation at $8000 \mathrm{rpm}$ for $2 \mathrm{~min}$ at 4 ${ }^{\circ} \mathrm{C}$ and washed thrice with $1 \mathrm{ml}$ homogenate buffer, then resuspended in $50 \mathrm{mM} \mathrm{NH}_{4} \mathrm{HCO}_{3}$ twice the volume of beads. After boiling, the supernatant was added $200 \mu \mathrm{l}$ chromatographic grade ethaol, blended and centrifugated at $12000 \mathrm{rpm}, 4{ }^{\circ} \mathrm{C}$ for $30 \mathrm{~min}$ to discard precipitation. The solution obtained was 
purified and concentrated with Amicon Ultra-0.5 ml Centrifugal Filter Units (Millipore), and detected by Analysis and Testing Center of Nanjing Medical University.

\section{Preperation of recombinant TREM2}

The plasmids encoding human TREM2, rat TREM2, mouse TREM2 as well as hTREM2(R47A), hTREM2(S65A), hTREM2(R77A) were purchesed from Public Protein/Plasmid Library. TREM2 expression for binding assay was performed as described previously[29]. In brief, TREM2 was expressed in freestyle 293F cells and purified using Ni-NTA resin and AKTA for further purification. The protein was stored in PBS and used to measure binding affinity rapidly.

\section{Microscale thermophoresis (MST)}

The above-mentioned obtained fusion proteins were labelled using Monolith His-Tag Labeling Kit (NanoTemper Technologies, Munich, Germany). The recombinant TREM2 and S1P were prepared at the concentration of $250 \mathrm{nM}$ and $2 \mathrm{mM}$, respectively. The binding affinity was detected with Monolith NT.115 (NanoTemper Technologies, Munich, Germany).

\section{Surface Plasmon Resonance (SPR)}

The obtained fusion proteins and S1P were prepared as described above. The fusion proteins were attached to Sensor chip NTA (Biacore). The binding affinity was detected with GE Biacore T200 (GE, USA).

\section{Apoptosis assay}

The TUNEL Apoptosis Assay Kit was purchased from KeyGEN bioTECH (Jiangsu, China) and performed following the manufacturer's instructions.

\section{Statistical analysis}

The obtained data are presented as mean \pm SEM of at least two independent experiments. The relationship between two factors was analyzed using Pearson correlation analysis and groups were compared using a two-way ANOVA with post hoc Bonferroni's multiple comparisons test. All of the data were analyzed with GraphPad Prism 6.0 software. A value of $p<0.05$ indicated that the difference was statistically significant.

\section{Results}

\section{FTY720 promoted microglial phagocytosis in ischemic stroke rats}

We first investigated the pro-phagocytic effects of FTY720 in ischemic rats via NeuN-labeled neuron, Iba1-labeled microglia and CD68-labeled phagocytic microglia. We found that supplement with FTY720, an analog of S1P after phosphorylation, could promote microglia to phagocytose debris indicating by the 
increased proportions of $\left(\mathrm{Iba}^{+}+\mathrm{NeuN}^{+}\right)$cells/total Iba ${ }^{+}$cells $(\mathrm{Fig} .1, \mathrm{~B}$ and $\mathrm{C}),\left(\mathrm{Iba}^{+}+\mathrm{NeuN}^{+}\right)$cells/total $\mathrm{NeuN}^{+}$cells (Fig. 1, B and D), CD68 ${ }^{+}$area per microglia (Fig. 1, E and F). We used two-photon microscope to further confirm that FTY720 significantly enhanced the microglial phagocytosis of neuronal debris appeared at $48 \mathrm{~h}$ after MCAO (Fig. 1G). These data demonstrate the pro-phagocytic function of FTY720.

\section{S1P or FTY720p enhances phagocytosis via TREM2 rather than S1PRs}

In order to demonstrate whether S1PRs mediate the pro-phagocytosis of S1P and FTY720 in microglia, we knocked down S1PR2 and S1PR4, the two major subtypes expressed on the microglia (Fig. S1A). The results showed that S1PR2 and S1PR4 knockdown did not affect the pro-phagocytosis of S1P (Fig. S1B and C), indicating certain receptor other than S1PRs should mediate microglial phagocytosis.

TREM2 is an important phagocytosis mediator that exclusively expressed on microglia in the CNS. Its endogenous ligand has not been found, but phospholipids was proposed as one of candidates. Since S1P shares structural similarities with phospholipids, we speculated that S1P might be an endogenous ligand for TREM2. Hence, we used computer simulation of molecular docking to predicate the potential interactions between S1P or FTY720p (FTY720p is structurally similar to S1P) and TREM2. The predicated results showed that human TREM2 (hTREM2) could bind to S1P and FTY720p via H-bonds at $47 R, 65 S$ and $77 R$, as well as via salt bridge at $47 R$ and $77 R$ (Fig. 2A).

Then, we confirmed the interaction between S1P/FTY720p and TREM2 via LC-MS/MS (Fig. 2B), Microscale Thermophoresis (MST) (Fig. 2, C, E and F) and Surface Plasmon Resonance (SPR) (Fig. 2D). S1P-binding affinity of human TREM2 (hTREM2), rat TREM2 (rTREM2) and mouse TREM2 (mTREM2) were $62.59 \pm 11.93 \mu \mathrm{M}, 56.80 \pm 13.96 \mu \mathrm{M}$ and $64.62 \pm 16.64 \mu \mathrm{M}$, respectively. Simultaneously, we examined the FTY720p-binding affinity of hTREM2, rTREM2 and mTREM2. The data showed that FTY720p had higher binding affinity to hTREM2, rTREM2 $(6.75 \pm 1.80 \mu \mathrm{M}, 7.17 \pm 2.21 \mu \mathrm{M}$, respectively), and relative lower affinity to mTREM2 $(72.60 \pm 21.02 \mu \mathrm{M})($ Fig. $2 \mathrm{H})$. However, results from MST showed that FTY720 failed to bind to hTREM2, suggesting phosphorylation was the precondition for FTY720 binding to TREM2 (Fig. 2G).

Furtherly, we constructed $\mathrm{CHO}$ cell expressing TREM2-DAP12 (T/D CHO cells, Fig. 3A) to identify the prophagocytic functions of S1P on TREM2-DAP12 complex. As shown in Fig. 3B, CHO cells did not phagocytose pHrodo Red zymosan bioparticles with or without S1P or LPS treatment. Importantly, S1P or LPS, increased phagocytosis of T/D CHO cells in a time-dependent pattern (Fig. 3, B and C), which was cancelled by phagocytosis inhibitor CytoD. Collectively, our data demonstrate that S1P or FTY720p functions as TREM2 ligand to enhance phagocytosis.

\section{R47, S65 and R77 are the crucial residues for the binding of S1P and TREM2}


To further determine the binding site, we induced the point mutation according to computer prediction data (Fig. 2I). R47A, S65A or R77A variants caused slightly lower affinity of S1P to hTREM2 (111.76 \pm $21.85 \mu \mathrm{M}, 93.92 \pm 21.35 \mu \mathrm{M}, 95.93 \pm 18.81 \mu \mathrm{M}$, respectively) (Fig. 2, J to L). However, R47A/S65A/R77A variant of hTREM2 remarkably lowered the binding affinity to $239.87 \pm 12.20 \mu \mathrm{M}$ (Fig. 2M). These data confirmed the prediction results by computer simulation of molecular docking, and revealed the importance roles of R47, S65 and R77 residues in the binding of S1P to TREM2.

\section{The pro-phagocytosis and neuroprotective effects of S1P/FTY720 in stroke are reduced when TREM2 was knockdown or knockout}

In order to further confirm the crucial roles of TREM2 in S1P/FTY720-induced microglial phagocytosis, we used TREM2 knockout (TREM2 ${ }^{-/-}$) mice to investigate the effect of FTY720. Consistant with previous results, FTY720 significantly promoted phagocytosis, as indicated by increased proportions of phagocytic microglia (Fig. 4, A and B) and ${\mathrm{CD} 68^{+}}^{+}$area per microglia (Fig. 4, A and C). However, the prophagocytosis effect of FTY720 was abolished in TREM2 ${ }^{-/-}$mice (Fig. 4, A to C). In vitro data showed similar results. We co-cultured normal or TREM2 knockdown microglia with neurons, followed by OGD/R treatment. Phagocytosis by normal microglia significantly increased at $5 \mathrm{~h}$ after reperfusion, peaked at $7 \mathrm{~h}$ and decreased thereafter (Fig. 4, D to G). S1P or FTY720 treatment dramatically promoted phagocytosis at $3 \mathrm{~h}$. However, TREM2 knockdown obviously inhibited the effect. These data highlight the importance of TREM2 in S1P/FTY720-induced pro-phagocytosis.

The expression of S1P in the penumbra of ischemic stroke rats were decreased at 24 and $48 \mathrm{~h}$ after reperfusion (Fig. 5A), indicating the insufficient S1P in acute phase of cerebral ischemia. FTY720 treatment could supplement the shortage (Fig. 5A), promote cellular debris clearance (Fig. 1 ) and thereby play the neuroprotective effects, indicated by decreased infarct volume (Fig. 5, B and C) and alleviated neurological deficits (Fig. 5D). However, the neuroprotection was not observed in TREM2 ${ }^{-/-}$mice treated with FTY720 after MCAO (Fig. 5E). We further assessed the protective effects of S1P/FTY720 via microglia-neuron co-culture system. At $24 \mathrm{~h}$ after OGD/R, S1P or FTY720 treatment maintained the length of longest neurite of neurons, which was significantly shortened when knockdown of TREM2 in microglia without affecting the number of neurites (Fig. 6, A to C). Consistently, S1P or FTY720 dramatically decreased apoptotic cells, which was abolished by TREM2 knockdown or CytoD (Fig. 6, D and E). These data show that TREM2 is pivotal in FTY720/S1P induced phagocytosis and protection.

Considering the significance of phagocytosis in hemorrhagic stroke, which is characterized by accumulated blood products like hemaglobin $(\mathrm{Hb})$ in the CNS, firstly, we investigated the effect of S1P on the microglial clearance of $\mathrm{Hb}$. After $4 \mathrm{~h}$ of $\mathrm{Hb}$ treatment, primary microglia showed nearly no $\mathrm{Hb}$ clearance. Microglia barely phagocytosed $\mathrm{Hb}$ when treated with low dose of S1P ( $250 \mathrm{nM})$, which is able to activate S1PRs[30]. However, high dose of S1P $(5 \mu \mathrm{M})$ significantly promoted the phagocytosis of $\mathrm{Hb}$, 
which was almost completely abolished by cytoD (Fig. S2A). In the hemorrhagic stroke mice, FTY720 treatment dramatically increased $\mathrm{Hb}$ clearance at $72 \mathrm{~h}$ after ICH (Fig. S2B), ameliorated injury as indicated by reduced hematoma volume (Fig. S2C and D) and lower neurological score (Fig. S2E). Taken together, our data suggest that S1P/FTY720 could promote phagocytosis and alleviated stroke-induced injury via TREM2.

\section{Discussion}

Recent findings reveal the importance of rapid clearance of cellular debris after ischemic stroke[31, 32]. In the present study, we reveal for the first time that S1P acts as a novel endogenous ligand of TREM2 to effectively promote microglial phagocytosis, and thereby play neuroprotective effects in stroke.

As is known, S1P can be secreted outside cells and activate S1PRs to regulate differentiation, survival, proliferation, angiogenesis and immune modulation[4, 5]. A few studies have suggested that S1P could regulate microglial phagocytosis[6, 7] via unknown mechanisms. We found that S1P and its analog FTY720 could enhance microglial phagocytosis. However, S1PR2 and S1PR4, main S1PRs expressed on microglia, did not participated in the pro-phagocytic function, indicating certain receptor other than S1PRs mediates the effect.

TREM2 is exclusively expressed on microglia in the brain whose endogenous ligand is still unknown. Since S1P structurally resembles phospholipids, we speculated the potential interaction between S1P and TREM2 to promote phagocytosis. Firstly, computer simulation of molecular docking predicated that S1P or FTY720p could bind to TREM2. For verification, LC-MS/MS, MST and SPR were performed and identified the affinity. The results showed the binding affinity of S1P to hTREM2, rTREM2 and mTREM2 were $62.59 \pm 11.93 \mu \mathrm{M}, 56.80 \pm 13.96 \mu \mathrm{M}$ and $64.62 \pm 16.64 \mu \mathrm{M}$, respectively. FTY720p has higher affinity to hTREM2 and rTREM2, but lower affinity to mTREM2. TREM2-DAP12 transfected CHO cells were used to further demonstrate the pro-phagocytic function of S1P via acting on TREM2. Furthermore, the point mutation analysis suggested that R47, S65 and R77 are the crucial residues for the binding of S1P and TREM2. Overall, our results reveal that S1P is a novel endogenous ligand for TREM2 to promote phagocytosis, and that S1P can be uses as a lead compound for modification to increase its affinity and effects.

Emerging evidence suggest that TREM2 is essential for disease associated microglia (DAM) $[33,34]$ and microglial neurodegenerative phenotype (MGnD)[35] transition, [36]. Whether S1P activates TREM2 to facilitate microglia transition to these phenotypes, and then promote phagocytosis deserves further investigation. The neuroprotection of FTY720 in MCAO models has been proved[37, 38]. However, the contribution of FTY720 induced cellular debris clearance to its protective effects has not been reported. We found that S1P in the penumbra decreased in the acute phase of ischemic stroke, accompanied by insufficient phagocytic function of microglia. Supplemented with FTY720 could significantly enhance microglial phagocytose. We further investigated the crucial role of TREM2 in mediating phagocytosis using TREM2 ${ }^{-/-}$mice and TREM2 knockdown microglia. As expected, the pro-phagocytic function of 
FTY720 and S1P was abolished in TREM2 ${ }^{-/-}$mice and TREM2-deficient microglia, resulting in reduced protective effect. These data confirm that S1P or FTY720 acts on TREM2 to induce phagocytosis and protection.

In addition to cerebral ischemia, phagocytosis also exerts critical role in hemorrhagic stroke. Rapid clearance of $\mathrm{Hb}$ should be protective in the hemorrhagic stroke. However, increased activity and expression of S1P-lyase occur after hemorrhage result in a reduction of 60\% in S1P level[39]. FTY720 treatment significantly reduced the hematoma volume and neurological deficit[40-42] which could not be abolished by blockage of central S1PRs [43]. Previous studies have uncovered that S1P forms a complex with $\mathrm{Hb}$ and promotes deoxy- $\mathrm{Hb}$ anchoring to the membrane[30]. On the basis of our findings, we supposed that FTY720 may function as S1P analog to mediate TREM2 recognition of $\mathrm{Hb}$ for phagocytosis. To prove the point, we investigated the $\mathrm{Hb}$ clearance by isolated microglia after $\mathrm{Hb}$ treatment. $\mathrm{Hb}$ was not be cleared by microglia cultured in normal culture medium or low dose of S1P (250 nM), but significantly be phagocytized by microglia treated with high dose of S1P (5 $\mu \mathrm{M})$. These data also suggested S1P-induced phagocytosis mediated by TREM2 rather than S1PRs. The prophagocytosis effect of FTY720 was further observed in the peri-hematoma following ICH.

Collectively, our results reveal for the first time that S1P is a novel endogenous ligand for TREM2. S1P or its analog FTY720p, binding to TREM2, promote microglial phagocytosis and exerts neuroprotection. Our findings provide a new lead compound for develop TREM2 agonist and a promising strategy for modulating microglia to treat ischemic stroke.

\section{Conclusion}

In summary, we reveal that S1P is a novel endougenous TREM2 ligand that promotes microglial phagocytosis and debris clearance. Expression of S1P decreases in the penumbra within $48 \mathrm{~h}$ after cerebral ischemia. Targeting TREM2-S1P interaction is a promising strategy to alleviate damaged and dead neurons induced damage, and to exert neuroprotection. These findings provide a new lead compound for developing TREM2 modulator.

\section{Abbreviations}




\begin{tabular}{ll} 
S1P & sphingosine 1-phosphate \\
\hline FTY720 & fingolimod \\
\hline FTY720p & fingolimod phosphate \\
\hline TREM2 & triggering receptor expressed on myeloid cells 2 \\
\hline MST & microscale thermophoresis \\
\hline SPR & surface plasmon resonance \\
\hline LC-MS/MS & liquid chromatography-tandem mass spectrometry \\
\hline MCAO & middle cerebral artery occlusion \\
\hline ICH & intracerebral hemorrhage \\
\hline DAM & disease associated microglia
\end{tabular}

\section{Declarations}

\section{Ethics approval and consent to participate}

The protocols were approved by the Institutional Animal Care and Use Committee of Nanjing Medical University.

\section{Consent for publication}

Not applicable.

\section{Availability of data and materials}

Results generated and analyzed during the current study are included in this article and its supplementary files.

\section{Competing interests}

The authors declare no competing interests.

\section{Funding}

The National Natural Science Foundation of China (Nos. 81973301 and 81773701), Jiangsu Key Research and Development Program (No. BE2017737), the Natural Science Foundation of the Jiangsu 
Higher Education Institutions of China (No. 18KJA310004), the Major Project of Nanjing Medical University (No. NMUD2018008) and the Postgraduate Research and Practice Innovation Program of Jiangsu Province (Nos. KYCX19_1121 and KYCX20_1417).

\section{Authors' contributions}

Ruo-Bing Guo and Wei Guo performed the animal experiments. Juan Ji and Yu-Qin Sun performed all in vitro experiments. Xi-Peng Wang performed the neurological deficit scoring and TTC staining. Zhen-Yu Cai, Xin-Xin Huang and Jin Yang synthesized and purified the TREM2 protein. Teng-Fei Xue and Juan Ji conceived the study and reseach design, analyzed the data and performed statistical analyses. Teng-Fei Xue perfomed binding assays and wrote the manuscript. Hong Cheng, Hai-Bin Shi and Xiu-Lan Sun supervised all aspects of the study. All authors critically read approved the final manuscript.

\section{Acknowledgements}

We appreciate Analysis and Testing Center of Nanjing Medical University for performing LC-MS/MS to determine the TREM2-S1P interaction.

\section{References}

1. Spiegel S, Milstien S: The Outs and the Ins of sphingosine-1-phosphate in Immunity. Nat Rev Immunol 2011, 11:403-415.

2. Lincheng Zhang, Yutong Dong, Yiran Wang, Wenhui Hu, Shiwu Dong, Chen Y: Sphingosine-1phosphate (S1P) Receptors: Promising Drug Targets for Treating Bone-Related Diseases. J Cell Mol Med 2020, 24:4389-4401.

3. Zhenyan Jiang, Zhang $\mathrm{H}$ : Molecular Mechanism of S1P Binding and Activation of the S1P1 Receptor. J Chem Inf Model 2019, 59:4402-4412.

4. Aynaz Mihanfar, Hamid Reza Nejabati, Amir Fattahi, etc: The role of sphingosine 1 phosphate in coronary artery disease and ischemia reperfusion injury. Journal of Cellular Physiology 2019, 234:2083-2094.

5. Zohaib Raza, Uzma Saleem, Naureen Z: Sphingosine 1-phosphate signaling in ischemia and reperfusion injury. Prostaglandins Other Lipid Mediat 2020, 149:106436.

6. Luo B, Gan W, Liu Z, etc: Erythropoeitin Signaling in Macrophages Promotes Dying Cell Clearance and Immune Tolerance. Immunity 2016, 44:287-302.

7. Hai B. Tran, Hubertus Jersmann, Tung Thanh Truong, etc: Disrupted epithelial/macrophage crosstalk via Spinster homologue 2-mediated S1P signaling may drive defective macrophage phagocytic function in COPD. PLoS One 2017, 12:e0179577. 
8. Agnieszka Paradowska-Gorycka, Jurkowska M: Structure, expression pattern and biological activity of molecular complex TREM-2/DAP12. Human Immunology 2013, 74:730-737.

9. Athena Sudom, Santosh Talreja, Jean Danao, etc: Molecular basis for the loss-of-function effects of the Alzheimer's disease-associated R47H variant of the immune receptor TREM2. The Journal of Biological Chemistry 2018, 293:12634-12646.

10. Carmen Mecca, lleana Giambanco, Rosario Donato, Arcuri C: Microglia and Aging: The Role of the TREM2-DAP12 and CX3CL1-CX3CR1 Axes. International Journal of Molecular Sciences 2018, 19:pii: E318.

11. Meghan M. Painter, Yuka Atagi, Chia-Chen Liu, etc: TREM2 in CNS homeostasis and neurodegenerative disease. Molecular Neurodegeneration 2015, 10.

12. Poliani PL, Wang Y, Fontana E, Robinette ML, Yamanishi Y, Gilfillan S, Colonna M: TREM2 sustains microglial expansion during aging and response to demyelination. The Journal of Clinical Investigation 2015, 125:2161-2170.

13. Elsa-Noah N'Diaye, Catherine S. Branda, Steven S. Branda, Lisette Nevarez, Marco Colonna, etc: TREM-2 (triggering receptor expressed on myeloid cells 2 ) is a phagocytic receptor for bacteria. The Journal of Cell Biology 2009, 184:215-223.

14. Michael R. Daws, Paul M. Sullam, Eréne C. Niemi, Thomas T. Chen, Nadia K. Tchao, Seaman WE: Pattern Recognition by TREM-2: Binding of Anionic Ligands. The Journal of Immunology 2003, 171:594-599.

15. David N. Quan, Morris D. Cooper, Jamie L. Potter, Melissa H. Roberts, Hui Cheng, Jarvis GA: TREM-2 Binds to Lipooligosaccharides of Neisseria gonorrhoeae and is Expressed on Reproductive Tract Epithelial Cells. Mucosal Immunology 2008, 1:229-238.

16. Yaming Wang, Marina Cella, Kaitlin Mallinson, etc: TREM2 lipid sensing sustains microglia response in an Alzheimer's disease model. Cell 2015, 160:1061-1071.

17. Grysiewicz RA, Thomas K, Pandey DK: Epidemiology of Ischemic and Hemorrhagic Stroke: Incidence, Prevalence, Mortality, and Risk Factors. Neurol Clin 2008, 26:871-895.

18. Emelia J. Benjamin, Michael J. Blaha, Stephanie E. Chiuve, etc: Heart Disease and Stroke Statistics2017 Update: a report from the American Heart Association. Circulation 2017, 135:e146-e603.

19. Anthony S. Kim, Johnston SC: Temporal and Geographic Trends in the Global Stroke Epidemic. Stroke 2013, 44:S123-S125.

20. Ulrich Dirnagl, Costantino ladecola, Moskowitz MA: Pathobiology of ischaemic stroke: an integrated view. Trends Neurosci 1999, 22:391-397.

21. Suresh L. Mehta, Namratta Manhas, Raghubir R: Molecular targets in cerebral ischemia for developing novel therapeutics. Brain Res Rev 2007, 54:34-66.

22. Frederic Geissmann, Markus G. Manz, Steffen Jung, Michael H. Sieweke, Miriam Merad, Ley K: Development of monocytes, macrophages and dendritic cells. Science 2010, 327:656-661. 
23. Jun Tsuyama, Akari Nakamura, Hiroaki Ooboshi, Akihiko Yoshimura, Shichita T: Pivotal role of innate myeloid cells in cerebral post-ischemic sterile

inflammation. Seminars in Immunopathology 2018, 40:523-538.

24. Hankey GJ: Stroke. Lancet 2017, 389:641-654.

25. Xi G, Keep RF, Hoff JT: Mechanisms of brain injury after intracerebral haemorrhage. Lancet Neurol 2006, 5:53-63.

26. Juan Ji, Juan Wang, Jin Yang, etc: The Intra-nuclear SphK2-S1P Axis Facilitates M1-to-M2 Shift of Microglia via Suppressing HDAC1-Mediated KLF4 Deacetylation. Front Immuno/ 2019, 10:1241.

27. Jialiang Wei, Ming Wang, Chaohui Jing, Richard F. Keep, Ya Hua, Xi G: Multinucleated Giant Cells in Experimental Intracerebral Hemorrhage. Trans/ Stroke Res 2020.

28. Wayne Clark, Lisa Gunion-Rinker, Nikola Lessov, Hazel K: Citicoline Treatment for Experimental Intracerebral Hemorrhage in Mice. Stroke 1998, 29:2136-2140.

29. Kober DL, Wanhainen KM, Johnson BM, etc: Preparation, crystallization, and preliminary crystallographic analysis of wild-type and mutant human TREM-2 ectodomains linked to neurodegenerative and inflammatory diseases. Protein Expr Purif 2014, 96:32-38.

30. Kaiqi Sun, Yujin Zhang, Angelo D'Alessandro, Travis Nemkov, Anren Song, Hongyu Wu, Hong Liu, Morayo Adebiyi, Aji Huang, Yuan E. Wen, et al: Sphingosine-1-phosphate promotes erythrocyte glycolysis and oxygen release for adaptation to high-altitude hypoxia. Nature Communications 2016, 7:12086.

31. Lilly M Winfree, Sean D Speese, Logan MA: Protein phosphatase 4 coordinates glial membrane recruitment and phagocytic clearance of degenerating axons in Drosophila. Cell Death and Disease 2017, 8:e2623.

32. Yamaguchi A, Jitsuishi T, Hozumi T: Temporal expression profiling of DAMPs-related genes revealed the biphasic post-ischemic inflammation in the experimental stroke model. Mol Brain 2020, 13:57.

33. Aleksandra, Deczkowska, Hadas Keren-Shaul, Assaf Weiner, Marco Colonna, Michal Schwartz, Amit I: Disease-Associated Microglia: A Universal Immune Sensor of Neurodegeneration. Cell 2018, 173:1073-1081.

34. Hadas Keren-Shaul, Amit Spinrad, Assaf Weiner, etc: A Unique Microglia Type Associated with Restricting Development of Alzheimer's Disease. Cel/ 2017, 169:1276-1290.

35. Susanne Krasemann, Charlotte Madore, Ron Cialic, etc: The TREM2-APOE Pathway Drives the Transcriptional Phenotype of Dysfunctional Microglia in Neurodegenerative Diseases. Immunity 2017, 47:566-581.

36. Alicia A Nugent, Karin Lin, Bettina van Lengerich, etc: TREM2 Regulates Microglial Cholesterol Metabolism upon Chronic Phagocytic Challenge. Neuron 2020, 105:837-854.

37. Maryam Nazaria, Somaye Keshavarza, Ali Rafati, Mohammad Reza Namavar, Haghani M: Fingolimod (FTY720) Improves Hippocampal Synaptic Plasticity and Memory Deficit in Rats 
Following Focal Cerebral Ischemia. Brain Res Bull 2016, 124:95-102.

38. Yu Hasegawa, Hidenori Suzuki, Orhan Altay, William Rollanda, Zhang JH: Role of the Sphingosine Metabolism Pathway on Neurons Against Experimental Cerebral Ischemia in Rats. Trans/ Stroke Res 2013, 4:524-532.

39. Fernando D Testai, Hao-Liang Xu, John Kilkus, Vidyani Suryadevara, Irina Gorshkova, Evgeny Berdyshev, Dale A Pelligrino, Dawson Dawson: Changes in the Metabolism of Sphingolipids after Subarachnoid Hemorrhage. J Neurosci Res 2015, 93:796-805.

40. Yu-Jing Li, Guo-Qiang Chang, Yuanchu Liu, Ye Gong, Chunsheng Yang, Kristofer Wood, Fu-Dong Shi, Ying Fu, Yan Y: Fingolimod alters inflammatory mediators and vascular permeability in intracerebral hemorrhage. Neurosci Bull 2015, 31:755-762.

41. Lia R, Venkatb P, Chopp M: RP001 hydrochloride improves neurological outcome after subarachnoid hemorrhage. J Neurol Sci 2019, 399:6-14.

42. Zhiyong Yang, Sisi Dong, Qiuyue Zheng, Lingling Zhang, Xinmei Tan, Jun Zou, Bingchun Yan, Chen Y: FTY720 attenuates iron deposition and glial responses in improving delayed lesion and long-term outcomes of collagenase-induced intracerebral hemorrhage. Brain Res 2019, 1718:91-102.

43. Yu Hasegawa, Ken Uekawa, Takayuki Kawano, Hidenori Suzuki, Kim-Mitsuyama S: Blockage of Central Sphingosine-1-phosphate Receptor does not Abolish the Protective Effect of FTY720 in Early Brain Injury after Experimental Subarachnoid Hemorrhage. Curr Drug Deliv 2017, 14:861-866.

\section{Figures}




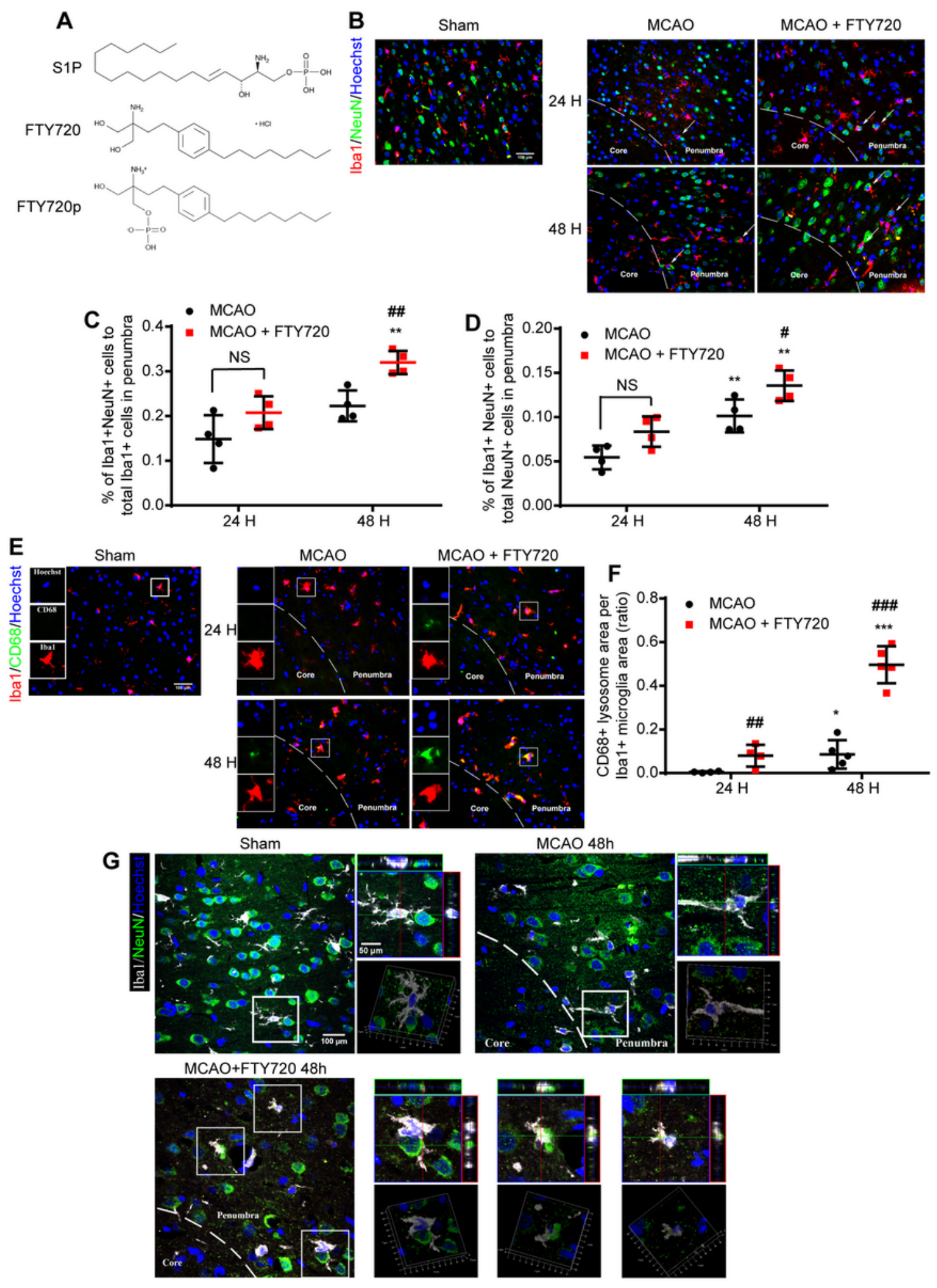

\section{Figure 1}

FTY720 promotes microglia phagocytosing neuronal debris in the penumbra of MCAO rats. (A) Chemical structure of S1P, FTY720 and FTY720p. (B) Immunofluorescence staining of NeuN+ neuronal debris (yellow) enwrapped by Iba1+ microglia (red) in MCAO group or MCAO + FTY720 group. The percentage of phagocytic microglia (C), phagocytosed neuronal debris (D) were analyzed; $n=4$ for each group, ${ }^{\star *} \mathrm{P}<$ 0.01 vs corresponding group at $24 \mathrm{~h}, \# \mathrm{P}<0.05$ and \#\#P $<0.01$ vs MCAO group. (E) Representative 
images of microglial CD68 expression. CD68 (green) was colocalized with Iba1 (red). Nuclei counterstained with Hoechst (blue) in MCAO group and MCAO + FTY720 group. (F) Quantification of CD68 expression, indicated by ratio of CD68+ area to Iba1+ area; $n=4-5$. ${ }^{*} P<0.05$ and ${ }^{* \star *} \mathrm{P}<0.001$ vs corresponding group at $24 \mathrm{~h}, \# \# \mathrm{P}<0.01$ and \#\#\#P $<0.001$ vs MCAO group. Values are presented as mean \pm SD and analyzed by two-way ANOVA and Tukey's test. (G) Microglial phagocytosis observed via Two-photon microscopy in the penumbra at $48 \mathrm{~h}$ after ischemia; $\mathrm{n}=3$ for each group.

A

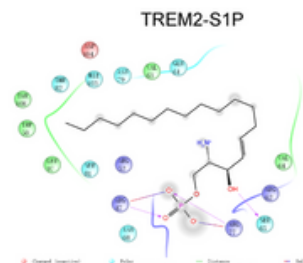

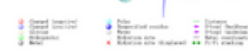
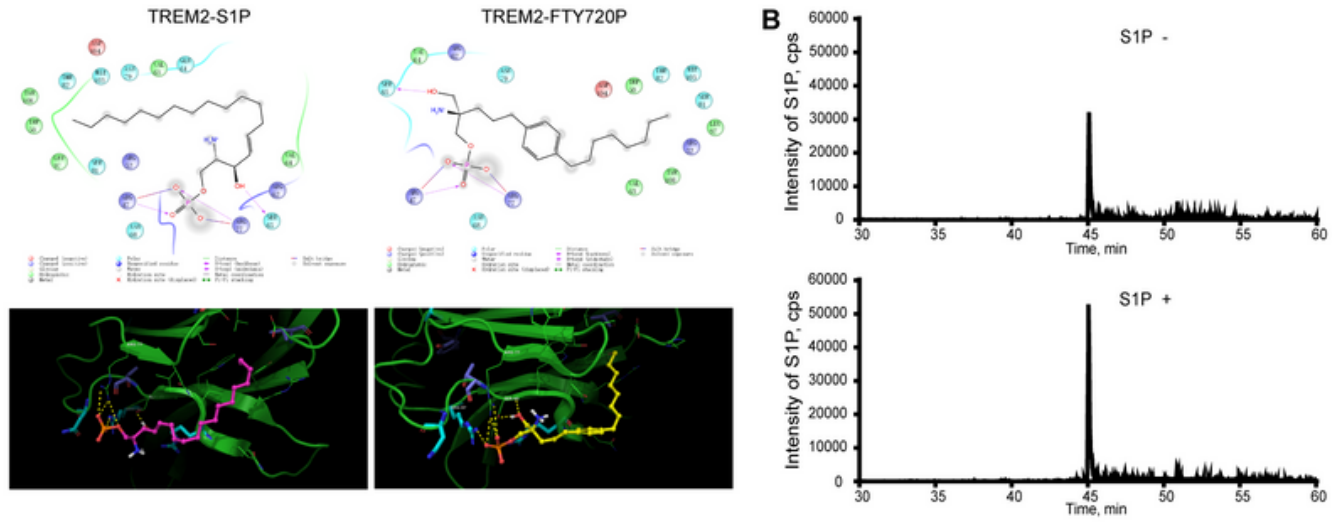

C

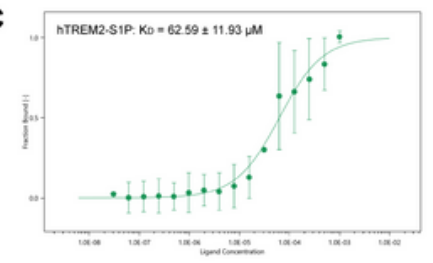

$\mathbf{E}$

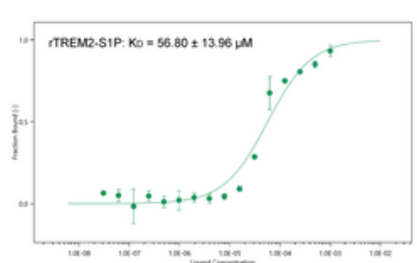

F

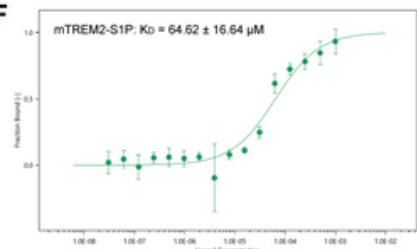

G

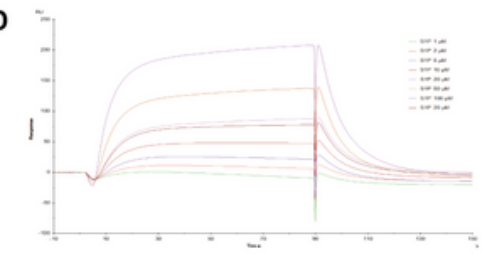

H
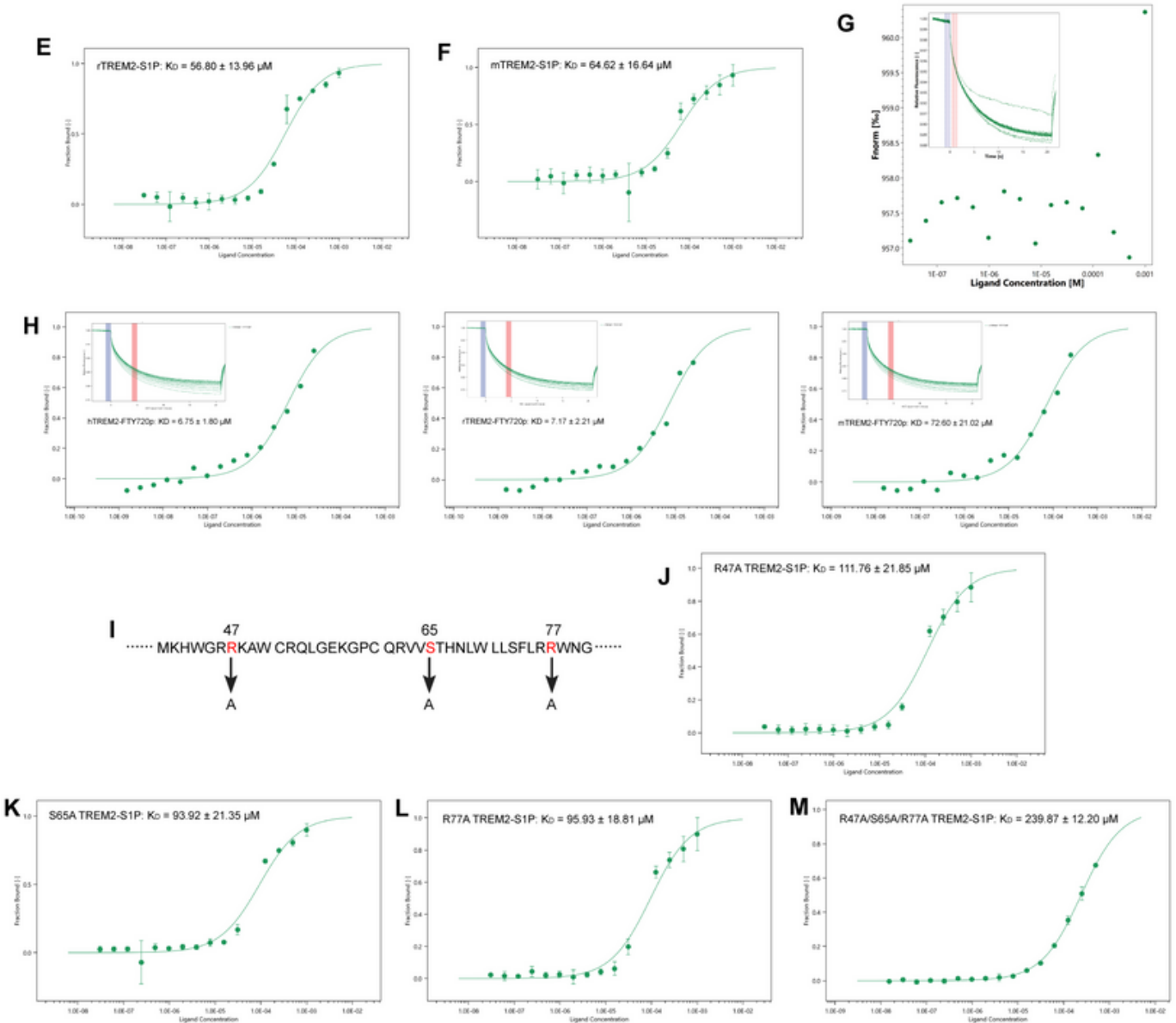

Figure 2 
S1P is an endogenous TREM2 ligand binding at R47, S65 and R77. (A) Molecular docking results showing the potential interaction between TREM2 and S1P or FTY720P. (B) LC-MS/MS spectra of TREM2 immunoprecipitated S1P of BV2 extract after normal culture or $1 \mathrm{~h} 20 \mu \mathrm{M} \mathrm{S1P}$ treatment. The binding of fluorescently labelled hTREM2 (C), rTREM2 (E), mTREM2 (F) to S1P is analysed with MST. S1P is titrated from $30.5 \mathrm{nM}$ to $1 \mathrm{mM}$. The change in the thermophoretic signal leads to a $\mathrm{KD}=62.59 \pm 11.93 \mu \mathrm{M}, 56.80$ $\pm 13.96 \mu \mathrm{M}, 64.62 \pm 16.64 \mu \mathrm{M}$, respectively. (D) The binding profiles of S1P to different concentrations of hTREM2 were generated by SPR assay. (G) MST result showing no interaction between hTREM2 and FTY720. (H) The binding of fluorescently labelled hTREM2, rTREM2, mTREM2 to FTY720p is analysed with MST. FTY720p is titrated from $1.53 \mathrm{nM}$ to $50 \mu \mathrm{M}$. The change in the thermophoretic signal leads to a $\mathrm{KD}=6.75 \pm 1.80 \mu \mathrm{M}, 7.17 \pm 2.21 \mu \mathrm{M}, 72.60 \pm 21.02 \mu \mathrm{M}$, respectively. (I) Schematic representing of site of point mutation induced to hTREM2. The binding of fluorescently labelled R47A TREM2 (J), S65A TREM2 (K), R77A TREM2 (L) and R47A/S65A/R77A TREM2 (M) to S1P is analysed with MST. S1P is titrated from $30.5 \mathrm{nM}$ to $1 \mathrm{mM}$. The change in the thermophoretic signal leads to a $\mathrm{KD}=111.76 \pm 21.85 \mu \mathrm{M}, 93.92$ $\pm 21.35 \mu \mathrm{M}, 95.93 \pm 18.81 \mu \mathrm{M}$ and $239.87 \pm 12.20 \mu \mathrm{M}$, respectively. 


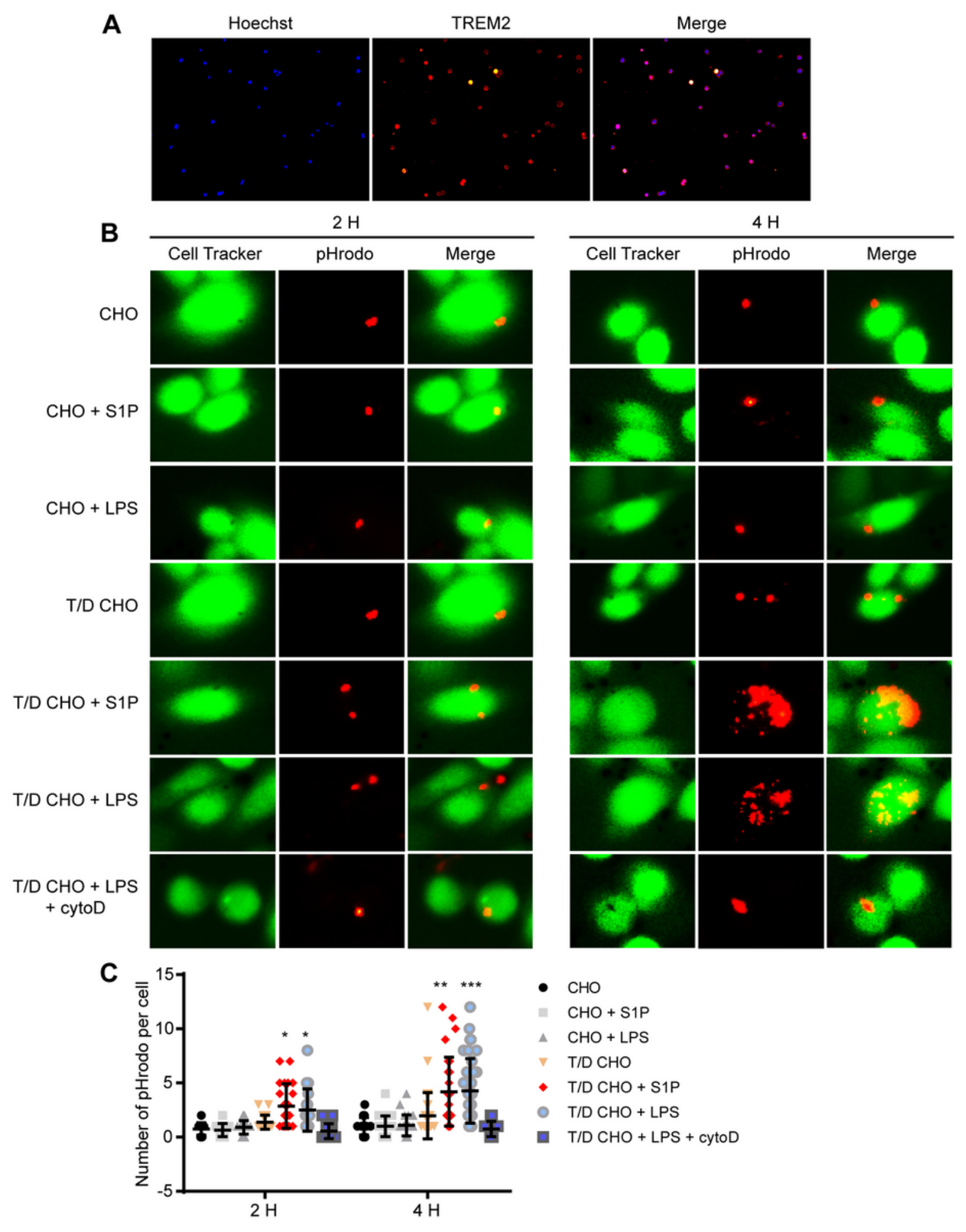

\section{Figure 3}

TREM2/DAP12 transfected $\mathrm{CHO}$ cells confirm that S1P increases phagocytosis via TREM2. (A) Immunofluorescence image confirming the successfully constructed TREM2/DAP12 transfected CHO cells. (B) Phagocytosis of pHrodo by transfected cells analyzed by fluorescence microscopy. CHO cells or TREM2/DAP12 cells were challenged with complete medium, $40 \mu \mathrm{M} \mathrm{S1P,} 40 \mu \mathrm{M} \mathrm{S1P}+2 \mu \mathrm{M}$ cytoD or 10 $\mu \mathrm{g} / \mathrm{ml}$ LPS, set as positive control, respectively. (C) Quantification of phagocytosed pHrodo in each cell 


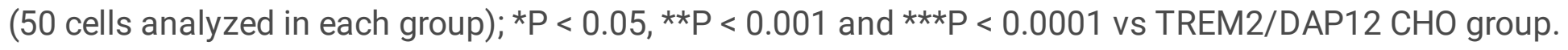
The graph represents the mean \pm SD and analyzed by t-test.

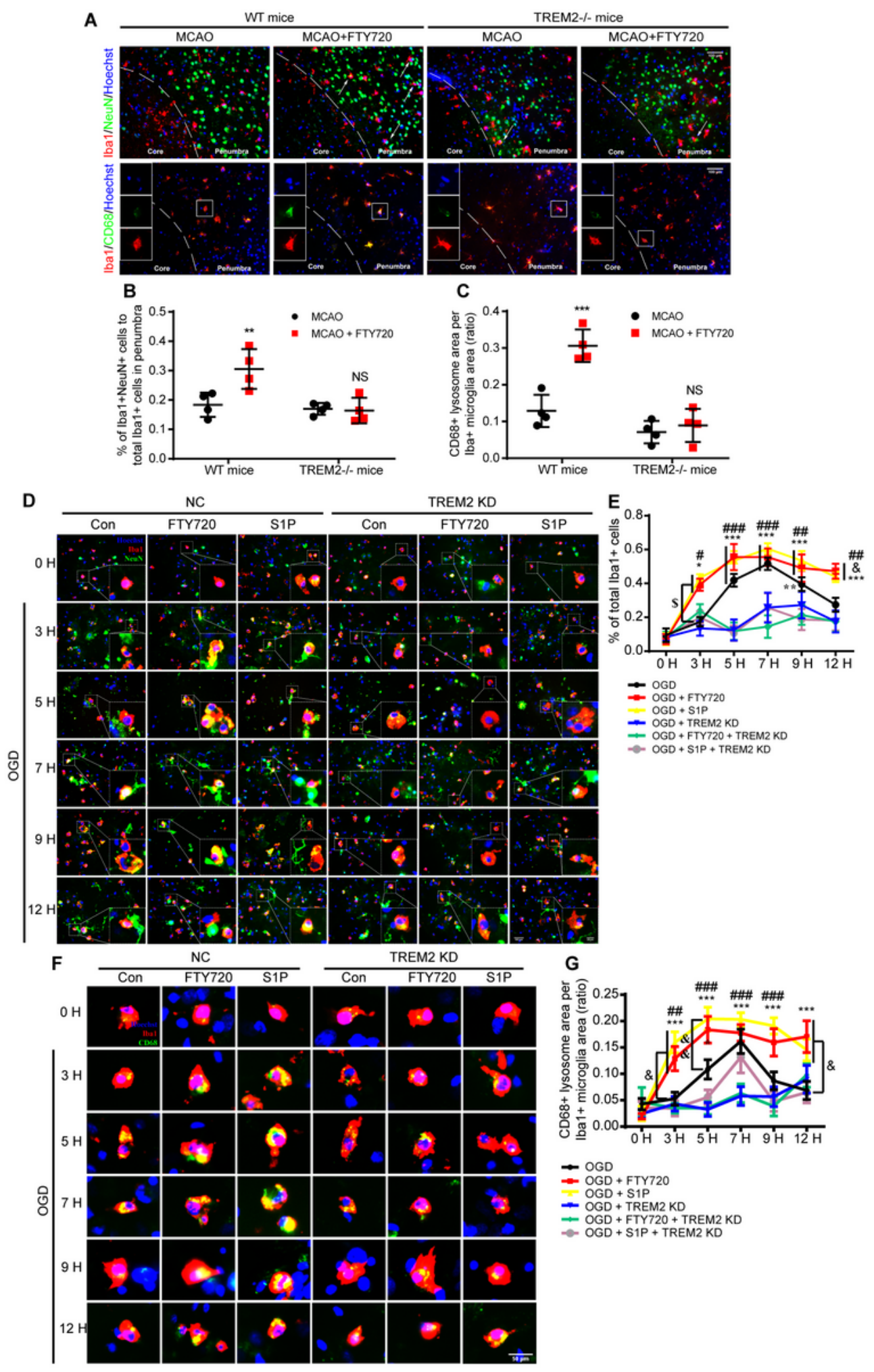

Figure 4

TREM2 deficiency abolishes the pro-phagocytic function of FTY720 and S1P. (A) Immunofluorescence staining of neuronal debris (yellow) enwrapped by microglia (red, upper row) or of microglial CD68 level (lower row) in WT or TREM2-/- MCAO mice with saline or FTY720 (1 mg/kg) treatment for $48 \mathrm{~h}$. The 
percentage of phagocytic microglia (B) and CD68 expression (C) were analyzed; $n=4$ for each group. (D) Immunofluorescence staining of NeuN (green), Iba1 (red) and Hoechst (blue) in NC or TREM2 KD microglia-neuron coculture system. Primary cells were treated with complete medium, $10 \mathrm{nM}$ S1P or FTY720 following OGD. The experiment underwent three independent replicates. (E) showed quantification of phagocytosing microglia; ${ }^{*} P<0.05$, ${ }^{*} \mathrm{P}<0.01$ and $* \star * P<0.001$ vs corresponding Con group, \#P $<0.05$, \#\#P $<0.01$ and \#\#\#P $<0.001$ vs corresponding TREM2 KD group, \&P $<0.05$ vs corresponding OGD group. (F) Immunofluorescence staining of CD68 (green), lba1 (red) and Hoechst (blue) in NC or TREM2 KD microglia-neuron coculture system. (G) Quantification of CD68 expression as ratio of CD68 area to Iba1 area; ${ }^{\star \star *} \mathrm{P}<0.001$ vs corresponding Con group, \#\#P<0.01 and \#\#\#P<0.001 vs corresponding TREM2 KD group, \&P $<0.05$ and \&\&P $<0.01$ vs corresponding OGD group. Values are expressed expressed as mean \pm SD and analyzed by two-way ANOVA and Tukey's test.
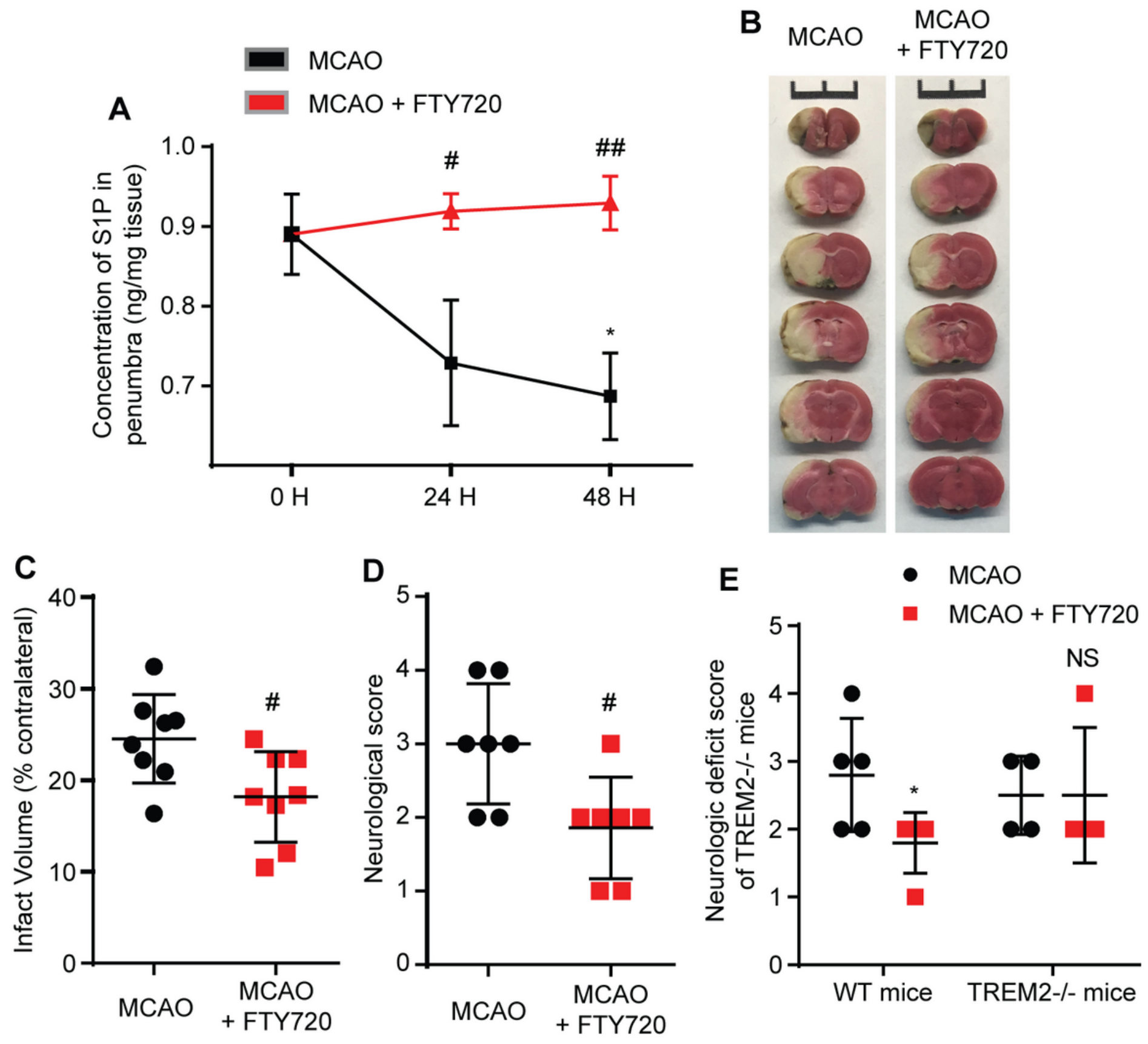


\section{Figure 5}

Shortage of S1P after ischemia and protection of FTY720 in ischemic stroke. (A) Dynamic variation of S1P level in the penumbra of MCAO rats measured by ELISA; $n=6, * P<0.05$ vs Sham group, \#P<0.05 and \#\#P $<0.01$ vs MCAO group. (B) TTC staining of brain sections from MCAO or MCAO + FTY720 rats and (C) Quantificative analysis of infarct volume; $n=8$, \#p $<0.05$ vs MCAO group. Neurological deficits via Bederson's scale scores were analyzed in rats $(D, n=8)$ and WT or TREM2-/- mice $(E, n \geq 4)$. Values are presented as mean \pm SD and analyzed by two-way ANOVA and Tukey's test or t-test.

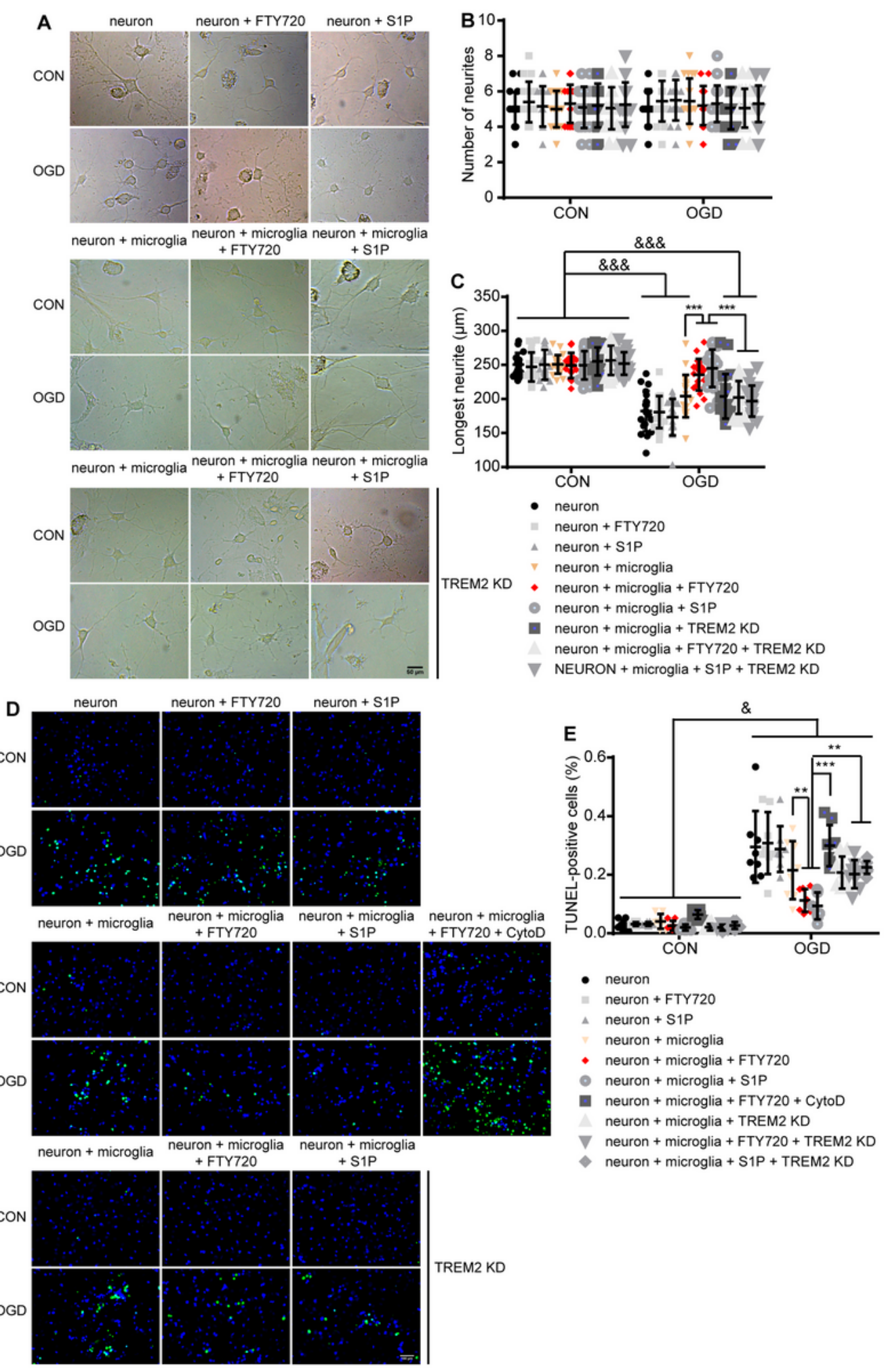




\section{Figure 6}

Phagocytosis enhancement via S1P-TREM2 interaction reduces neurite shortening and apoptotic cells. (A) Representative images showing neurons in normal neuron-microglia coculture or that at $24 \mathrm{~h}$ after OGD. The experiment underwent three independent replicates. Number of neurites (B) and lenghth of longest neurites (C) were analyzed; $\star \star \star \mathrm{P}<0.001$ as indicated, \&\&\&P $<0.001$ vs corresponding groups at $24 \mathrm{~h}$ after OGD. (D) Merged images of Hoechst (blue) and TUNEL (green) in the indicated groups. The experiment underwent three independent replicates. (E) Quantitative analysis of percentage of TUNEL positive cells. ${ }^{\star \star P} P<0.01$ and ${ }^{\star \star *} \mathrm{P}<0.001$ vs indicated groups, $\& \mathrm{P}<0.05$ vs corresponding groups at $24 \mathrm{~h}$ after OGD. Values are expressed as mean \pm SD and analyzed by two-way ANOVA and Tukey's test.

\section{Supplementary Files}

This is a list of supplementary files associated with this preprint. Click to download.

- Fig.S1.tif

- Fig.S2.tif 